\title{
New Primal-Dual Proximal Algorithm for Distributed Optimization
}

\author{
Puya Latafat, Lorenzo Stella, Panagiotis Patrinos
}

\begin{abstract}
We consider a network of agents, each with its own private cost consisting of the sum of two possibly nonsmooth convex functions, one of which is composed with a linear operator. At every iteration each agent performs local calculations and can only communicate with its neighbors. The goal is to minimize the aggregate of the private cost functions and reach a consensus over a graph. We propose a primal-dual algorithm based on Asymmetric Forward-Backward-Adjoint (AFBA), a new operator splitting technique introduced recently by two of the authors. Our algorithm includes the method of Chambolle and Pock as a special case and has linear convergence rate when the cost functions are piecewise linear-quadratic. We show that our distributed algorithm is easy to implement without the need to perform matrix inversions or inner loops. We demonstrate through computational experiments how selecting the parameter of our algorithm can lead to larger step sizes and yield better performance.
\end{abstract}

\section{INTRODUCTION}

In this paper we deal with the distributed solution of the following optimization problem:

$$
\underset{x \in \mathbb{R}^{n}}{\operatorname{minimize}} \sum_{i=1}^{N} f_{i}(x)+g_{i}\left(C_{i} x\right)
$$

where for $i=1, \ldots, N, C_{i}$ is a linear operator, $f_{i}$ and $g_{i}$ are proper closed convex and possibly nonsmooth functions. We further assume that the proximal mappings associated with $f_{i}$ and $g_{i}$ are efficiently computable [1]. In a more general case we can include another continuously differentiable term with Lipschitz-continuous gradient in (1) and use [2, Algorithm 3] that includes the algorithm of Vũ and Condat [3], [4] as special case. We do not pursue this here for clarity of exposition.

Problems of this form appear in several application fields. In a distributed model predictive control setting, $f_{i}$ can represent individual finite-horizon costs for each agent, $C_{i}$ model the linear dynamics of each agent and possibly coupling constraints that are split through the introduction of extra variables, and $g_{i}$ model state and input constraints.

In machine learning and statistics the $C_{i}$ are feature matrices and functions $g_{i}$ measure the fitting of a predicted model with the observed data, while the $f_{i}$ is regularization terms that enforces some prior knowledge in the solution (such as sparsity, or belonging to a certain constraint set).

P. Latafat is with IMT School for Advanced Studies Lucca, Piazza San Francesco 19, 55100 Lucca, Italy; Email: puya. latafateimtlucca.it

L. Stella and P. Patrinos are with the Department of Electrical Engineering (ESAT-STADIUS) and Optimization in Engineering Center (OPTEC), KU Leuven, Kasteelpark Arenberg 10, 3001 Leuven-Heverlee, Belgium; Emails: lorenzo.stella@esat.kuleuven.be, panos.patrinoseesat.kuleuven.be
For example if $g_{i}$ is the so-called hinge loss and $f_{i}=\frac{\lambda}{2}\|\cdot\|_{2}^{2}$, for some $\lambda>0$, then one recovers the standard SVM model. If instead $f_{i}=\lambda\|\cdot\|_{1}$ then one recovers the $\ell_{1}$-norm SVM problem [5].

Clearly problem (1) can be solved in a centralized fashion, when all the data of the problem (functions $f_{i}, g_{i}$ and matrices $C_{i}$, for all $\left.i \in\{1, \ldots, N\}\right)$ are available at one computing node. When this is the case one might formulate and solve the aggregated problem

$$
\underset{x \in \mathbb{R}^{n}}{\operatorname{minimize}} f(x)+g(C x),
$$

for which algorithms are available [2], [6], [7]. However, such a centralized approach is not realistic in many scenarios. For example, suppose that $g_{i}\left(C_{i} x\right)$ models least-squares terms and $C_{1}, \ldots, C_{N}$ are very large features matrices. Then collecting $C_{1}, \ldots, C_{N}$ into a single computer may be infeasible due to communication costs, or even worse they may not fit into the computer's memory. Furthermore, the exchange of such information may not be possible at all due to privacy issues.

Our goal is therefore to solve problem (1) in a distributed fashion. Specifically, we consider a connected network of $N$ computing agents, where the $i$-th agent is able to compute proximal mappings of $f_{i}, g_{i}$, and matrix vector products with $C_{i}$ (and its adjoint operator). We want all the agents to iteratively converge to a consensus solution to (1), and to do so by only exchanging variables among neighbouring nodes, i.e, no centralized computations (i.e., existence of a fusion center) are needed during the iterations.

To do so, we will propose a solution based on the recently introduced Asymmetric Forward-Backward-Adjoint (AFBA) splitting method [2]. This new splitting technique solves monotone inclusion problems involving three operators, however, in this work we will focus on a special case that involves two terms. Specifically, we develop a distributed algorithm which is based on a special case of AFBA applied to the monotone inclusion corresponding to the primal-dual optimality conditions of a suitable graph splitting of (1). Our algorithm involves a nonnegative parameter $\theta$ which serves as a tuning knob that allows to recover different algorithms. In particular, the algorithm of [6] is recovered in the special case when $\theta=2$. We demonstrate how tuning this parameter affects the stepsizes and ultimately the convergence rate of the algorithm.

Other algorithms have been proposed for solving problems similar to (1) in a distributed way. As a reference framework, 
all algorithms aim at solving in a distributed way the problem

$$
\underset{x \in \mathbb{R}^{n}}{\operatorname{minimize}} \sum_{i=1}^{N} F_{i}(x) \text {. }
$$

In [8] a distributed subgradient method is proposed, and in [9] this idea is extended to the projected subgradient method. More recently, several works focused on the use of ADMM for distributed optimization. In [10] the generic ADMM for consensus-type problems is illustrated. A drawback of this approach is that at every iteration the agents must solve a complicated subproblem that might require an inner iterative procedure. In [11] another formulation is given for the case where $F_{i}=f_{i}+g_{i}$, and only proximal mappings with respect to $f_{i}$ and $g_{i}$ are separately computed in each node. Still, when either $f_{i}$ or $g_{i}$ is not separable (such as when they are composed with linear operators) these are not trivial to compute and may require inner iterative procedures, or factorization of the data matrices involved. Moreover, in both [10], [11] a central node is required for accumulating each agents variables at every iteration, therefore these formulations lead to parallel algorithms rather than distributed. In [12] the optimal parameter selection for ADMM is discussed in the case of distributed quadratic programming problems. In [13][15], fully distributed algorithms based on ADMM proposed, assuming that the proximal mapping of $F_{i}$ is computable, which is impractical in many cases. In [16] the authors propose a variation of the Vũ-Condat algorithm [3], [4], having ADMM as a special case, and show its application to distributed optimization where $F_{i}=f_{i}+g_{i}$, but no composition with a linear operator is involved. Only proximal operations with respect to $f_{i}$ and $g_{i}$ and local exchange of variables (i.e., among neighboring nodes) is required, and the method is analyzed in an asynchronous setting.

In this paper we deal with the more general scenario of problem (1). The main features of our approach, that distinguish it from the related works mentioned above, are:

(i) We deal with $F_{i}$ that is the sum of two possibly nonsmooth functions one of which is composed with a linear operator.

(ii) Our algorithm only require local exchange of information, i.e., only neighboring nodes need to exchange local variables for the algorithms to proceed.

(iii) The iterations involve direct operations on the objective terms. Only evaluations of $\operatorname{prox}_{f_{i}}, \operatorname{prox}_{g_{i}^{\star}}$ and matrixvector products with $C_{i}$ and $C_{i}^{T}$ are involved. In particular, no inner subproblem needs to be solved iteratively by the computing agents, and no matrix inversions are required.

The paper is organized as follows. Section II is devoted to a formulation of problem (1) which is amenable to be solved in a distributed fashion by the proposed methods. In Section III we detail how the primal-dual algorithm in [2, Algorithm 6] together with an intelligent change of variables gives rise to distributed iterations. We then discuss implementation considerations and convergence properties. In Section IV we illustrate some numerical results for several values of the constant $\theta$, highlighting the improved performance for $\theta=1.5$.

\section{PROBLEM Formulation}

Consider problem (1) under the following assumptions:

Assumption 1. For $i=1, \ldots, N$ :

(i) $C_{i}: \mathbb{R}^{n} \rightarrow \mathbb{R}^{r_{i}}$ are linear operators.

(ii) $f_{i}: \mathbb{R}^{n} \rightarrow \overline{\mathbb{R}}, g_{i}: \mathbb{R}^{r_{i}} \rightarrow \overline{\mathbb{R}}$ are proper closed convex functions, where $\overline{\mathbb{R}}=\mathbb{R} \cup\{\infty\}$.

(iii) The set of minimizers of (1), denoted by $S^{\star}$, is nonempty.

We are interested in solving problem (1) in a distributed fashion. Specifically, let $G=(V, E)$ be an undirected graph over the vertex set $V=\{1, \ldots, N\}$ with edge set $E \subset$ $V \times V$. It is assumed that each node $i \in V$ is associated with a separate agent, and each agent maintains its own cost components $f_{i}, g_{i}, C_{i}$ which are assumed to be private, and its own opinion of the solution $x_{i} \in \mathbb{R}^{n}$. The graph imposes communication constraints over agents. In particular, agent $i$ can communicate directly only with its neighbors $j \in \mathcal{N}_{i}=$ $\{j \in V \mid(i, j) \in E\}$. We make the following assumption.

\section{Assumption 2. Graph $G$ is connected.}

With this assumption, we reformulate the problem as

$$
\begin{array}{cl}
\underset{\boldsymbol{x} \in \mathbb{R}^{N n}}{\operatorname{minimize}} & \sum_{i=1}^{N} f_{i}\left(x_{i}\right)+g_{i}\left(C_{i} x_{i}\right) \\
\text { subject to } & x_{i}=x_{j} \quad(i, j) \in E
\end{array}
$$

where $\boldsymbol{x}=\left(x_{1}, \ldots, x_{N}\right)$. Associate any orientation to the unordered edge set $E$. Let $M=|E|$ and $B \in \mathbb{R}^{N \times M}$ be the oriented node-arc incidence matrix, where each column is associated with an edge $(i, j) \in E$ and has +1 and -1 in the $i$-th and $j$-th entry, respectively. Notice that the sum of each column of $B$ is equal to 0 . Let $d_{i}$ denote the degree of a given vertex, that is, the number of vertices that are adjacent to it. We have $B B^{\top}=\mathcal{L} \in \mathbb{R}^{N \times N}$, where $\mathcal{L}$ is the graph Laplacian of $G$, i.e.,

$$
\mathcal{L}_{i j}= \begin{cases}d_{i} & \text { if } i=j \\ -1 & \text { if } i \neq j \text { and node } i \text { is adjacent to node } j \\ 0 & \text { otherwise. }\end{cases}
$$

Constraints $x_{i}=x_{j},(i, j) \in E$ can be written in compact form as $A \boldsymbol{x}=0$, where $A=B^{\top} \otimes I_{n} \in \mathbb{R}^{M n \times N n}$. Therefore, the problem is expressed as

$$
\underset{\boldsymbol{x} \in \mathbb{R}^{N n}}{\operatorname{minimize}} \sum_{i=1}^{N} f_{i}\left(x_{i}\right)+g_{i}\left(C_{i} x_{i}\right)+\delta_{\{0\}}(A \boldsymbol{x}),
$$

where $\delta_{X}$ denotes the indicator function of a closed nonempty convex set, $X$. The dual problem is:

$$
\underset{\substack{y_{i} \in \mathbb{R}^{r_{i}} \\ w \in \mathbb{R}^{M n}}}{\operatorname{minimize}} \sum_{i=1}^{N} f_{i}^{*}\left(-A_{i}^{\top} w-C_{i}^{\top} y_{i}\right)+g_{i}^{*}\left(y_{i}\right),
$$

where $q^{*}$ denotes the Fenchel conjugate of a function $q$ and $A_{i} \in \mathbb{R}^{M n \times n}$ are the block columns of $A$. Let $\partial q$ denote 
the subdifferential of a convex function $q$. The primal-dual optimality conditions are

$$
\begin{cases}0 \in \partial f_{i}\left(x_{i}\right)+C_{i}^{\top} y_{i}+A_{i}^{\top} w, & i=1, \ldots, N \\ C_{i} x_{i} \in \partial g_{i}^{*}\left(y_{i}\right) & i=1, \ldots, N \\ \sum_{i=1}^{N} A_{i} x_{i}=0, & \end{cases}
$$

where $w \in \mathbb{R}^{M n}, y_{i} \in \mathbb{R}^{r_{i}}$, for $i=1, \ldots, N$. The following condition will be assumed throughout the rest of the paper.

Assumption 3. There exist $x_{i} \in \operatorname{ridom} f_{i}$ such that $C_{i} x_{i} \in$ ridom $g_{i}, i=1, \ldots, N$ and $\sum_{i=1}^{N} A_{i} x_{i}=0^{1}$.

This assumption guarantees that the set of solutions to (4) is nonempty (see [17, Proposition 4.3(iii)]). If $\left(\boldsymbol{x}^{\star}, \boldsymbol{y}^{\star}, w^{\star}\right)$ is a solution to (4), then $x^{\star}$ is a solution to the primal problem (2) and $\left(\boldsymbol{y}^{\star}, w^{\star}\right)$ to its dual (3).

\section{Distributed Primal-Dual Algorithms}

In this section we provide the main distributed algorithm that is based on Asymmetric Forward-Backward-Adjoint (AFBA), a new operator splitting technique introduced recently [2]. This special case belongs to the class of primaldual algorithms. The convergence results include both the primal and dual variables and are based on [2, Propositions 5.4]). However, the convergence analysis here focuses on the primal variables for clarity of exposition, with the understanding that a similar error measure holds for the dual variables.

Our distributed algorithm consists of two phases, a local phase and the phase in which each agent interacts with its neighbors according to the constraints imposed by the communication graph. Each iteration has the advantage of only requiring local matrix-vector products and proximal updates. Specifically, each agent performs 2 matrix-vector products per iteration and transmits a vector of dimension $n$ to its neighbors.

Before continuing we recall the definition of Moreau's proximal mapping. Let $U$ be a symmetric positive-definite matrix. The proximal mapping of a proper closed convex function $f: \mathbb{R}^{n} \rightarrow \overline{\mathbb{R}}$ relative to $\|\cdot\|_{U}$ is defined by

$$
\operatorname{prox}_{f}^{U}(x)=\underset{z \in \mathbb{R}^{n}}{\operatorname{argmin}} f(z)+\frac{1}{2}\|x-z\|_{U}^{2},
$$

and when the superscript $U$ is omitted the same definition applies with respect to the canonical norm.

Let $\boldsymbol{u}=(\boldsymbol{x}, \boldsymbol{v})$ where $\boldsymbol{v}=(\boldsymbol{y}, w)$ and $\boldsymbol{y}=\left(y_{1}, \ldots, y_{N}\right)$. The optimality conditions in (4), can be written in the form of the following monotone inclusion:

$$
0 \in D \boldsymbol{u}+M \boldsymbol{u}
$$

with

$$
D(\boldsymbol{x}, \boldsymbol{y}, w)=\left(\partial \boldsymbol{f}(\boldsymbol{x}), \partial \boldsymbol{g}^{*}(\boldsymbol{y}), 0\right)
$$

\footnotetext{
${ }^{1} \operatorname{dom} f$ denotes the domain of function $f$ and $\operatorname{ri} C$ is the relative interior of the set $C$.
}

and

$$
M=\left[\begin{array}{ccc}
0 & \boldsymbol{C}^{\top} & A^{\top} \\
-\boldsymbol{C} & 0 & 0 \\
-A & 0 & 0
\end{array}\right],
$$

where $\boldsymbol{f}(\boldsymbol{x})=\sum_{i=1}^{k} f_{i}\left(x_{i}\right), \boldsymbol{g}^{*}(\boldsymbol{y})=\sum_{i=1}^{k} g_{i}^{*}\left(y_{i}\right)$, $\boldsymbol{C}=\operatorname{blkdiag}\left(C_{1}, \ldots, C_{N}\right)$. Notice that $A x=\sum_{i=1}^{k} A_{i} x_{i}$, $A^{\top} w=\left(A_{1}^{\top} w, \ldots, A_{N}^{\top} w\right)$. The operator $D+M$ is maximally monotone [18, Proposition 20.23, Corollary 24.4(i)].

Monotone inclusion (5), i.e., the primal-dual optimality conditions (4), is solved by applying [2, Algorithm 6]. This results in the following iteration:

$$
\begin{aligned}
\boldsymbol{x}^{k+1} & =\operatorname{prox}_{f}^{\Sigma}\left(\boldsymbol{x}^{k}-\Sigma C^{\top} \boldsymbol{y}^{k}-\Sigma A^{\top} w^{k}\right) \\
\overline{\boldsymbol{y}}^{k} & =\operatorname{prox}_{g^{*}}^{\Gamma}\left(\boldsymbol{y}^{k}+\Gamma C\left(\theta \boldsymbol{x}^{k+1}+(1-\theta) \boldsymbol{x}^{k}\right)\right) \\
\bar{w}^{k} & =w^{k}+\Pi A\left(\theta \boldsymbol{x}^{k+1}+(1-\theta) \boldsymbol{x}^{k}\right) \\
\boldsymbol{y}^{k+1} & =\overline{\boldsymbol{y}}^{k}+(2-\theta) \Gamma C\left(\boldsymbol{x}^{k+1}-\boldsymbol{x}^{k}\right) \\
w^{k+1} & =\bar{w}^{k}+(2-\theta) \Pi A\left(\boldsymbol{x}^{k+1}-\boldsymbol{x}^{k}\right)
\end{aligned}
$$

where matrices $\Sigma, \Gamma, \Pi$ play the rule of stepsizes and are assumed to be positive definite. The iteration (7) can not be implemented in a distributed fashion because the dual vector $w$ consists of $M$ blocks corresponding to the edges. The key idea that allows distributed computations is to introduce the sequence

$$
\left(\rho_{i}^{k}\right)_{k \in \mathbb{N}}=\left(A_{i}^{\top} w^{k}\right)_{k \in \mathbb{N}}, \quad \text { for } \quad i=1, \ldots, N .
$$

This transformation replaces the stacked edge vector $w^{k}$ with corresponding node vectors $\rho_{i}$. More compactly, letting $\boldsymbol{\rho}^{k}=$ $\left(\rho_{1}^{k}, \ldots, \rho_{N}^{k}\right)$, it follows from (7c) and (7e) that

$$
\boldsymbol{\rho}^{k+1}=\boldsymbol{\rho}^{k}+A^{\top} \Pi A\left(2 \boldsymbol{x}^{k+1}-\boldsymbol{x}^{k}\right),
$$

where $A^{\top} \Pi A$ is the weighted graph Laplacian. Since $w^{k}$ in (7a) appear as $A^{\top} w^{k}$ we can rewrite the iteration:

$$
\begin{aligned}
\boldsymbol{x}^{k+1} & =\operatorname{prox}_{f}^{\Sigma}\left(\boldsymbol{x}^{k}-\Sigma C^{\top} \boldsymbol{y}^{k}-\Sigma \boldsymbol{\rho}^{k}\right) \\
\overline{\boldsymbol{y}}^{k} & =\operatorname{prox}_{g^{*}}^{\Gamma}\left(\boldsymbol{y}^{k}+\Gamma C\left(\theta \boldsymbol{x}^{k+1}+(1-\theta) \boldsymbol{x}^{k}\right)\right) \\
\boldsymbol{y}^{k+1} & =\overline{\boldsymbol{y}}^{k}+(2-\theta) \Gamma C\left(\boldsymbol{x}^{k+1}-\boldsymbol{x}^{k}\right) \\
\boldsymbol{\rho}^{k+1} & =\boldsymbol{\rho}^{k}+A^{\top} \Pi A\left(2 \boldsymbol{x}^{k+1}-\boldsymbol{x}^{k}\right)
\end{aligned}
$$

Set

$$
\begin{aligned}
& \Sigma=\operatorname{blkdiag}\left(\sigma_{1} I_{n}, \ldots, \sigma_{N} I_{n}\right), \\
& \Gamma=\operatorname{blkdiag}\left(\tau_{1} I_{r_{1}}, \ldots, \tau_{N} I_{r_{N}}\right), \\
& \Pi=\operatorname{blkdiag}\left(\pi_{1} I_{n}, \ldots, \pi_{M} I_{n}\right),
\end{aligned}
$$

where $\sigma_{i}>0, \tau_{i}>0$ for $i=1, \ldots, N$ and $\pi_{l}>0$ for $l=1, \ldots, M$. Consider a bijective mapping between $l=$ $1, \ldots, M$ and unordered pairs $(i, j) \in E$ such that $\kappa_{i, j}=$ $\kappa_{j, i}=\pi_{l}$. Notice that $\pi_{l}$ for $l=1, \ldots, M$ are step sizes to be selected by the algorithm and can be viewed as weights for the edges. Thus, iteration (7) gives rise to our distributed algorithm: 


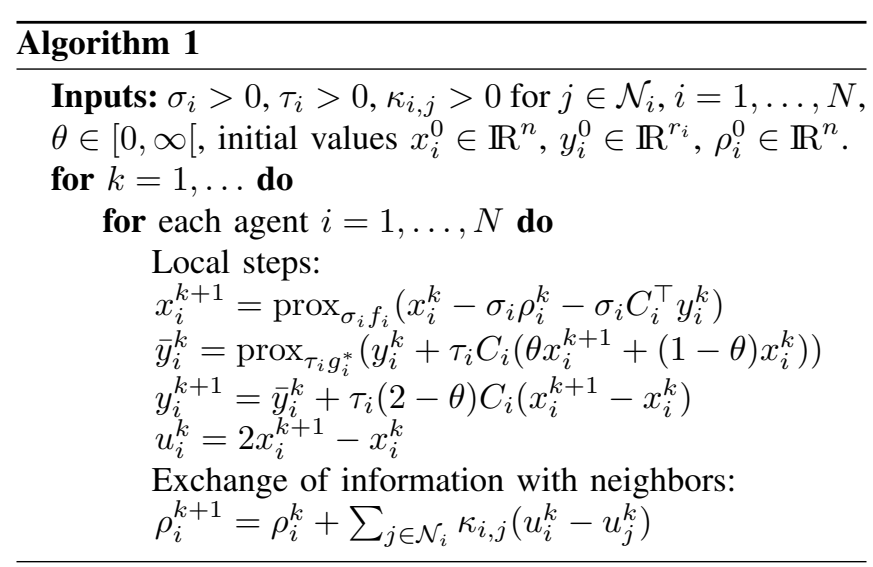

Notice that each agent $i$ only requires $u_{j}^{k} \in \mathbb{R}^{n}$ for $j \in$ $\mathcal{N}_{i}$ during the communication phase. Before proceeding with convergence results, we define the following for simplicity of notation:

$$
\begin{aligned}
\bar{\sigma} & =\max \left\{\sigma_{1}, \ldots, \sigma_{N}\right\}, \\
\bar{\tau} & =\max \left\{\tau_{1}, \ldots, \tau_{N}, \pi_{1}, \ldots, \pi_{M}\right\}, \\
L & =\mathcal{L} \otimes I_{n}+\boldsymbol{C}^{\top} \boldsymbol{C}, \text { where } \mathcal{L} \text { is the graph Laplacian. }
\end{aligned}
$$

It must be noted that the results in this section only provide choices of parameters that are sufficient for convergence. They can be selected much less conservatively by formulating and solving sufficient conditions that they must satisfy as linear matrix inequalities (LMIs). Due to lack of space we do not pursue this direction further, instead we plan to consider it in an extended version of this work.

Theorem 1. Let Assumptions 2 and 3 hold true. Consider the sequence $\left(\boldsymbol{x}^{k}\right)_{k \in \mathbb{N}}=\left(x_{1}^{k}, \ldots, x_{N}^{k}\right)_{k \in \mathbb{N}}$ generated by Algorithm 1. Assume the maximum stepsizes, i.e., $\bar{\sigma}$ and $\bar{\tau}$ defined above, are positive and satisfy

$$
\bar{\sigma}^{-1}-\bar{\tau}\left(\theta^{2}-3 \theta+3\right)\|L\|>0,
$$

for a fixed value of $\theta \in\left[0, \infty\left[\right.\right.$. Then the sequence $\left(\boldsymbol{x}^{k}\right)_{k \in \mathbb{N}}$ converges to $\left(x^{\star}, \ldots, x^{\star}\right)$ for some $x^{\star} \in S^{\star}$. Furthermore, if $\theta=2$ the strict inequality (10) is replaced with $\bar{\sigma}^{-1}-$ $\bar{\tau}\|L\| \geq 0$.

Proof. Algorithm 1 is an implementation of [2, Algorithm 6]. Thus convergence of $\left(\boldsymbol{x}^{k}\right)_{k \in \mathbb{N}}$ to a solution of (2) is implied by [2, Proposition 5.4]. Combining this with Assumption 2 yields the result. Notice that in that work the step sizes are assumed to be scalars for simplicity. It is straightforward to adapt the result to the case of diagonal matrices.

In Algorithm 1 when $\theta=2$, we recover the algorithm of Chambolle and Pock [6]. One important observation is that the term $\theta^{2}-3 \theta+3$ in (10) is always positive and achieves its minimum at $\theta=1.5$. This is a choice of interest for us since it results in larger stepsizes, $\sigma_{i}, \tau_{i}, \kappa_{i, j}$, and consequently better performance as we observe in numerical simulations.

Next, we provide easily verifiable conditions for $f_{i}$ and $g_{i}$, under which linear convergence of the iterates can be established. We remark that these are just sufficient and certainly less conservative conditions can be provided but are omitted for clarity of exposition. Let us first recall the following definitions from [19], [20]:

Definition 1 (Piecewise Linear-Quadratic). A function $f$ : $\mathbb{R}^{n} \rightarrow \overline{\mathbb{R}}$ is called piecewise linear-quadratic (PLQ) if it's domain can be represented as union of finitely many polyhedral sets, relative to each of which $f(x)$ is given by an expression of the form $\frac{1}{2} x^{\top} Q x+d^{\top} x+c$, for some $c \in \mathbb{R}$, $d \in \mathbb{R}^{n}$, and $D \in \mathbb{R}^{n \times n}$.

The class of piecewise linear-quadratic functions has been much studied and has many desirable properties (see [19, Chapter 10 and 11]). Many practical applications involve PLQ functions such as quadratic function, $\|\cdot\|_{1}$, indicator of polyhedral sets, hinge loss, etc. Thus, the $R$-linear convergence rate that we establish in Theorem 2 holds for a wide range of problems encountered in control, machine learning and signal processing.

Definition 2 (Metric subregularity). A set-valued mapping $F: \mathbb{R}^{n} \rightrightarrows \mathbb{R}^{n}$ is metrically subregular at $z$ for $z^{\prime}$ if $\left(z, z^{\prime}\right) \in$ gra $F$ and there exists $\eta \in[0, \infty[$, a neighborhood $\mathcal{U}$ of $z$ and $\mathcal{V}$ of $z^{\prime}$ such that

$$
d\left(x, F^{-1} z^{\prime}\right) \leq \eta d\left(z^{\prime}, F x \cap \mathcal{V}\right) \text { for all } x \in \mathcal{U},
$$

where gra $F=\{(x, u) \mid u \in F x\}$ and $d(\cdot, X)$ denotes the distance from set $X$.

Theorem 2. Consider Algorithm 1 under the assumptions of Theorem 1. Assume $f_{i}$ and $g_{i}$ for $i=1, \ldots, N$, are linear piecewise quadratic functions. Then the set valued mapping $T=D+M$ is metrically subregular at any $z$ for any $z^{\prime}$ provided that $\left(z, z^{\prime}\right) \in$ gra $T$. Furthermore, the sequence $\left(\boldsymbol{x}^{k}\right)_{k \in \mathbb{N}}$ converges $R$-linearly ${ }^{2}$ to $\left(x^{\star}, \ldots, x^{\star}\right)$ for some $x^{\star} \in S^{\star}$.

Proof. Function $\boldsymbol{f}(\boldsymbol{x})=\sum_{i=1}^{k} f_{i}\left(x_{i}\right)$ is piecewise linearquadratic since $f_{i}$ are assumed to be PLQ. Similarly it follows from [19, Theorem 11.14 (b)] that $\boldsymbol{g}^{*}$ is linear piecewise quadratic. The subgradient mapping of a proper closed convex PLQ function is piecewise polyhedral, i.e. its graph is the union of finitely many polyhedral sets [19, Proposition 12.30 (b)]. This shows that $D$ defined in (6) is piecewise polyhedral. Since the image of a polyhedral under affine transformation remains piecewise polyhedral, and $M$ is a linear operator, graph of $T=D+M$ is piecewise polyhedral. Consequently, its inverse $T^{-1}$ is piecewise polyhedral. Thus by [20, Proposition 3H.1] the mapping $T^{-1}$ is calm at any $z^{\prime}$ for any $z$ satisfying $\left(z^{\prime}, z\right) \in \operatorname{gra} T^{-1}$. This is equivalent to the metric subregularity characterization of the operator $T$ [20, Theorem 3H.3]. The second part of the proof follows directly by noting that [2, Algorithm 6] used to derive Algorithm 1 is a special case of [2, Algorithm 1]. Therefore linear convergence follows from first part of the

\footnotetext{
${ }^{2}$ The sequence $\left(x_{n}\right)_{n \in \mathbb{N}}$ converges to $x^{\star} R$-linearly if there is a sequence of nonnegative scalars $\left(v_{n}\right)_{n \in \mathbb{N}}$ such that $\left\|x_{n}-x^{\star}\right\| \leq v_{n}$ and $\left(v_{n}\right)_{n \in \mathbb{N}}$ converges $Q$-linearly ${ }^{3}$ to zero.

${ }^{3}$ The sequence $\left(x_{n}\right)_{n \in \mathbb{N}}$ converges to $x^{\star} Q$-linearly with $Q$-factor given by $\sigma \in] 0,1\left[\right.$, if for $n$ sufficiently large $\left\|x_{n+1}-x^{\star}\right\| \leq \sigma\left\|x_{n}-x^{\star}\right\|$ holds.
} 
proof together with [2, Theorem 3.3]. The aforementioned theorem guarantees linear convergence for the stacked vector $\boldsymbol{u}$ in (5), however, here we consider the primal variables only.

\section{A. Special Case}

Consider the following problem

$$
\underset{x \in \mathbb{R}^{n}}{\operatorname{minimize}} \sum_{i=1}^{N} f_{i}(x),
$$

where $f_{i}: \mathbb{R}^{n} \rightarrow \overline{\mathbb{R}}$ for $i=1, \ldots, N$ are proper closed convex functions. This is a special case of (1) when $g_{i} \circ C_{i} \equiv$ 0 . Since functions $g_{i}$ are absent, the dual variables $y_{i}$ in Algorithm 1 vanish and for any choice of $\theta$ the algorithm reduces to:

$$
\begin{aligned}
x_{i}^{k+1} & =\operatorname{prox}_{\sigma_{i} f_{i}}\left(x_{i}^{k}-\sigma_{i} \rho_{i}^{k}\right) \\
u_{i}^{k} & =2 x_{i}^{k+1}-x_{i}^{k} \\
\rho_{i}^{k+1} & =\rho_{i}^{k}+\sum_{j \in \mathcal{N}_{i}} \kappa_{i, j}\left(u_{i}^{k}-u_{j}^{k}\right) .
\end{aligned}
$$

Thus setting $\theta=1.5$ in (10) to maximize the stepsizes yields $\bar{\sigma}^{-1}-\frac{3 \bar{\tau}}{4}\|\mathcal{L}\|>0$, where $\mathcal{L}$ is the graph Laplacian.

\section{Numerical Simulations}

We now illustrate experimental results obtained by applying the proposed algorithm to the following problem:

$$
\text { minimize } \lambda\|x\|_{1}+\sum_{i=1}^{N} \frac{1}{2}\left\|D_{i} x-d_{i}\right\|_{2}^{2}
$$

for a positive parameter $\lambda$. This is the $\ell_{1}$ regularized leastsquares problem. Problem (13) is of the form (1) if we set for $i=1, \ldots, N$

$$
\begin{aligned}
f_{i}(x) & =\frac{\lambda}{N}\|x\|_{1}, \\
g_{i}(z) & =\frac{1}{2}\left\|z-d_{i}\right\|_{2}^{2}, \\
C_{i} & =D_{i}
\end{aligned}
$$

where $D_{i} \in \mathbb{R}^{m_{i} \times n}, d_{i} \in \mathbb{R}^{m_{i}}$. For the experiments we used graphs of $N=50$ computing agents, generated randomly according to the Erdős-Renyi model, with parameter $p=0.05$. In the experiments we used $n=500$ and generated $D_{i}$ randomly with normally distributed entries, with $m_{i}=50$ for all $i=1, \ldots, N$. Then we generated vector $d_{i}$ starting from a known solution for the problem and ensuring $\lambda<0.1\left\|\sum_{i}^{N} D_{i}^{\top} d_{i}\right\|_{\infty}$.

For the stepsize parameters we set $\sigma_{i}=\bar{\sigma}, \tau_{i}=\bar{\tau}$, for all $i=1, \ldots, N$, and $\kappa_{i, j}=\kappa_{j, i}=\bar{\tau}$ for all edges $(i, j) \in E$, such that (10) is satisfied. In order to have a fair comparison we selected $\bar{\sigma}=\alpha /\|L\|$ and $\bar{\tau}=0.99 /\left(\alpha\left(\theta^{2}-3 \theta+3\right)\right)$ with $\alpha=20$ which was set empirically based on better performance of all the algorithms.

The results are illustrated in Figure 1, for several values of $\theta$, where the distribution of the number of communication rounds required by the algorithms to reach a relative error of $10^{-6}$ is reported. In Figure 2 the convergence of algorithms is illustrated in one of the instances. It should be noted that

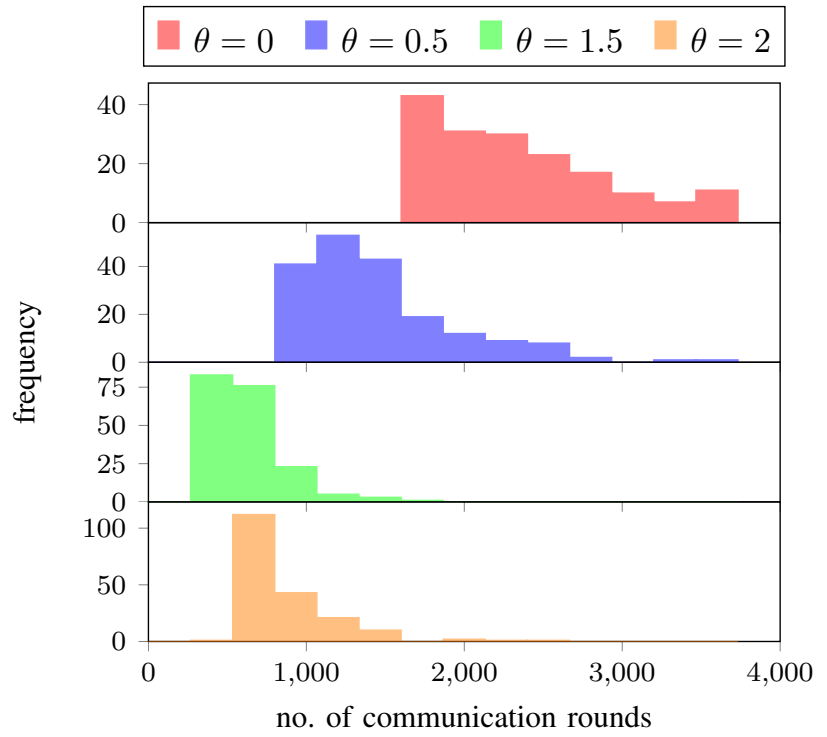

Fig. 1: Distribution of the number of communication rounds required by the algorithms to achieve a relative error of $10^{-6}$, for fixed data and 200 randomly generated ErdősRenyi graphs, with parameter $p=0.05$.

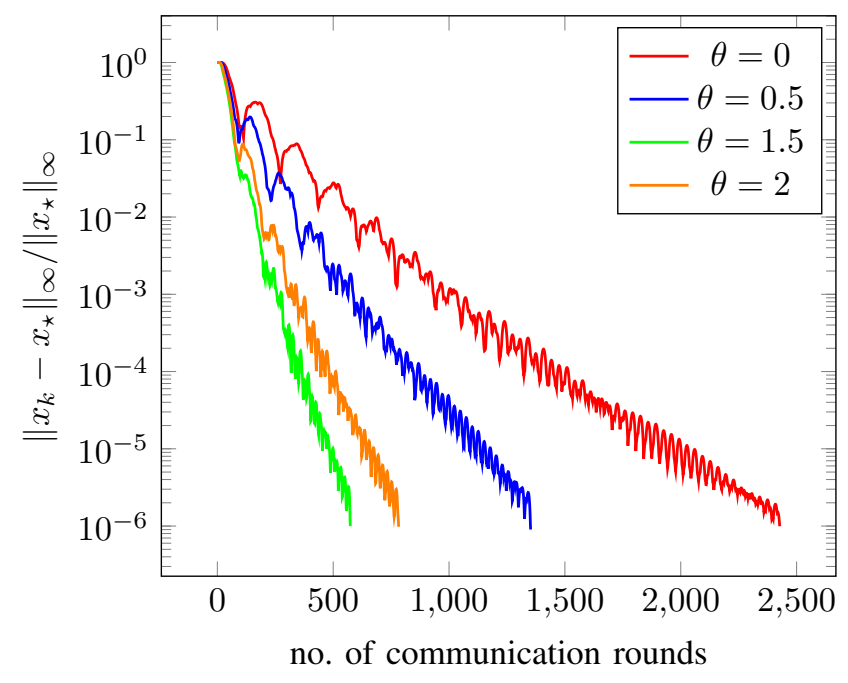

Fig. 2: Convergence of the relative error for the algorithms, in one of the considered instances.

the algorithm of Chambolle and Pock, that corresponds to $\theta=2$, is generally slower than the case $\theta=1.5$. This is mainly due to the larger stepsize parameters guaranteed by Theorem 1.

\section{COnClusions}

In this paper we illustrated how the recently proposed Asymmetric Forward-Backward-Adjoint splitting method (AFBA) can be used for solving distributed optimization problems where a set of $N$ computing agents, connected in a graph, need to minimize the sum of $N$ functions. The resulting Algorithm 1 only involves local exchange of variables (i.e., among neighboring nodes) and therefore no central authority is required to coordinate them. Moreover, 
the single nodes only require direct computations of the objective terms, and do not need to perform inner iterations and matrix inversions. Numerical experiments highlight that Algorithm 1 performs generally better when the parameter $\theta$ is set equal to 1.5 in order to achieve the largest stepsizes. Future investigations on this topic include the study of how the topological structure of the graph underlying the problem affects the convergence rate of the proposed methods, as well as problem preconditioning in a distributed fashion. Developing asynchronous versions of the algorithm is another important future research direction.

\section{REFERENCES}

[1] P. L. Combettes and I.-C. Pesquet, "Proximal splitting methods in signal processing," in Fixed-point algorithms for inverse problems in science and engineering. Springer, 2011, pp. 185-212.

[2] P. Latafat and P. Patrinos, "Asymmetric forward-backward-adjoint splitting for solving monotone inclusions involving three operators," arXiv preprint arXiv:1602.08729, 2016.

[3] B. C. Vũ, "A splitting algorithm for dual monotone inclusions involving cocoercive operators," Advances in Computational Mathematics, vol. 38 , no. 3, pp. 667-681, 2013.

[4] L. Condat, "A primal-dual splitting method for convex optimization involving Lipschitzian, proximable and linear composite terms," Journal of Optimization Theory and Applications, vol. 158, no. 2, pp. 460-479, 2013.

[5] J. Zhu, S. Rosset, T. Hastie, and R. Tibshirani, "1-norm support vector machines," Advances in neural information processing systems, vol. 16, no. 1, pp. 49-56, 2004.

[6] A. Chambolle and T. Pock, "A first-order primal-dual algorithm for convex problems with applications to imaging," Journal of Mathematical Imaging and Vision, vol. 40, no. 1, pp. 120-145, 2011.

[7] L. M. Briceño-Arias and P. L. Combettes, "A monotone + skew splitting model for composite monotone inclusions in duality," SIAM Journal on Optimization, vol. 21, no. 4, pp. 1230-1250, 2011.

[8] A. Nedić and A. Ozdaglar, "Distributed subgradient methods for multiagent optimization," IEEE Transactions on Automatic Control, vol. 54, no. 1, pp. 48-61, 2009.

[9] J. C. Duchi, A. Agarwal, and M. J. Wainwright, "Dual averaging for distributed optimization: convergence analysis and network scaling," IEEE Transactions on Automatic control, vol. 57, no. 3, pp. 592-606, 2012.

[10] S. Boyd, N. Parikh, E. Chu, B. Peleato, and J. Eckstein, "Distributed Optimization and Statistical Learning via the Alternating Direction Method of Multipliers," Foundations and Trends in Machine Learning, vol. 3, no. 1, pp. 1-122, 2010.

[11] N. Parikh and S. Boyd, "Block splitting for distributed optimization," Mathematical Programming Computation, vol. 6, no. 1, pp. 77-102, 2014.

[12] A. Teixeira, E. Ghadimi, I. Shames, H. Sandberg, and M. Johansson, "Optimal scaling of the ADMM algorithm for distributed quadratic programming," in IEEE 52nd Annual Conference on Decision and Control (CDC), 2013, pp. 6868-6873.

[13] E. Wei and A. Ozdaglar, "Distributed alternating direction method of multipliers," in IEEE 51 st Annual Conference on Decision and Control (CDC), 2012, pp. 5445-5450.

[14] — , "On the $O(1 / k)$ convergence of asynchronous distributed alternating direction method of multipliers," in IEEE Global Conference on Signal and Information Processing (GlobalSIP), 2013 IEEE, 2013, pp. $551-554$.

[15] A. Makhdoumi and A. Ozdaglar, "Broadcast-based distributed alternating direction method of multipliers," in 52nd Annual Allerton Conference on Communication, Control, and Computing (Allerton). IEEE, 2014, pp. 270-277.

[16] P. Bianchi and W. Hachem, "A primal-dual algorithm for distributed optimization," in IEEE 53rd Annual Conference on Decision and Control (CDC), Dec 2014, pp. 4240-4245.

[17] P. L. Combettes and J.-C. Pesquet, "Primal-dual splitting algorithm for solving inclusions with mixtures of composite, Lipschitzian, and parallel-sum type monotone operators," Set-Valued and variational analysis, vol. 20, no. 2, pp. 307-330, 2012.
[18] H. H. Bauschke and P. L. Combettes, Convex analysis and monotone operator theory in Hilbert spaces. Springer Science \& Business Media, 2011.

[19] R. T. Rockafellar and R. J.-B. Wets, Variational analysis. Springer Science \& Business Media, 2009, vol. 317.

[20] A. L. Dontchev and R. T. Rockafellar, "Implicit functions and solution mappings," Springer Monographs in Mathematics. Springer, vol. 208, 2009. 